\title{
Towards a Novel Way to Predict Deficits After a Brain Lesion: A Stroke Example
}

\author{
Georgios A. Klados \\ School of Electrical and Computer \\ Engineering Technical University of \\ Crete, Chania, Greece \\ gklados@isc.tuc.gr \\ Antonio Fratini \\ Dept. of Biomedical Engineering \\ School of Life and Health Sciences \\ Aston University, Birmingham, \\ United Kingdom \\ a.fratini@aston.ac.uk
}

\author{
Michalis Zervakis \\ School of Electrical and Computer \\ Engineering Technical University of \\ Crete, Chania, Greece \\ michalis@display.tuc.gr
}

Manousos A. Klados

Dept. of Biomedical Engineering

School of Life and Health Sciences,

Aston University, Birmingham,

United Kingdom

m.klados@aston.ac.uk

\author{
Rosalia Dacosta-Aguayo \\ Dept. of Psychiatry and Clinical \\ Psychobiology, Universitat de \\ Barcelona, Barcelona, Spain \\ rdacostaa@gmail.com
}

\begin{abstract}
Many studies have addressed the relations between different human brain regions and their role in cognitive, motor and sensory functions in patients that have suffered a brain lesion (stroke, traumatic brain injury, tissue removal). Nowadays, it is well established that the brain works as a network and the symptoms in a person are a combination of the direct impact of the lesion in a single region and its connectivity with other healthy brain regions. The aim of the present study is the development of a user-friendly desktop application to predict the induced cognitive deficits in patients who have suffered a brain lesion. The herein presented application is based on Neurosynth platform, and takes as an input a MRI mask that describes a lesion. Then our software exploits the knowledge that already exists in Neurosynth platform, so as to predict the potential deficits by grouping the Neurosynth's terms that have increased $Z$ scores with our mask. In addition, we have embedded two types of visualization methods: One to present the slices of the brain mask and another to show the $3 D$ volume of the mask into $3 D$ semitransparent human brain. The added value of the presented application is that it may give us a clue about which mechanisms are probably affected by a lesion in a specific region, while in the future it could provide neurosurgeons with insightful knowledge helping them in the plannification of a forthcoming surgical procedure. The proposed software was tested on 7 stroke patients, predicting accurately the $91 \%$ of the measured deficits found during a neuropsychological assessment.
\end{abstract} stroke

Index Terms-MRI, lesion, deficit prediction, visualization,

\section{INTRODUCTION}

The brain is a complex system in which a balance between specialization and integration is needed in order to get access to higher cognitive functions [1]. Whereas specialization takes place in circumscribed areas and has to be with the perception of the stimuli, the integration of the information provided by the stimuli needs the communication of different anatomical areas which not only give sense to what we perceive but allow our adaptation to our continuous changing environment [1].

The number of publications focused on the identification of different neuroimaging biomarkers for clinical diagnosis has increased exponentially during the last decade [1]. With the development of new neuroimaging modalities able to report different characteristics of brain tissue, we have the opportunity to combine them and apply different mathematical models in order to better understand and predict individual deficits related to brain lesions.
Actually, the ability to make such combinations is on the hands of professionals that have the skill to make such combinations and extract the most interesting features that can better predict and characterize cognitive and functional outcomes. However, other professionals with no such expertise are still looking at different acquisitions separately, in the best-case scenario, losing the opportunity to predict, with high accuracy.

Neurosynth (http://neurosynth.org/), is a known platform for "large scale automated synthesis of functional magnetic resonance imaging (fMRI)" that combines text mining, meta-analysis, and machine learning techniques to generate probabilistic mappings between cognitive and neural states. Thus, it performs feature-based meta-analysis based on statistical maps from 14371 fMRI studies [2]. Briefly, Neurosynth, extract key terms from the text of the analyzed fMRI studies and links them with the statistical maps that are presented in the current study. Then, given a voxel, it can tell you how strongly this voxel is linked with each one of the key-terms included in Neurosynth's database.

Therefore, our main idea is to exploit the term-toactivation mapping that Neurosynth provide us, with the aim to build a tool that can predict the potential deficits induced by a brain lesion. One problem towards this direction is that the terms used in Neurosynth's dictionary are either overlapping with other terms with a very close meaning (i.e., accuracy, accurate, accurately; act, action, acts, actions), the use terms their meaning is not straightforward (i.e., ad, add), or the use of anatomical terms that are useless for our purposes, while the key-term database contain words that are widely used during writing a manuscript.

The aforementioned problem can be solved by grouping the overlapping terms, by deleting anatomical useless terms, and by removing all the terms that are not related to neurophysiology. A question that arises when thinking about grouping those terms, is how we can combine the Zscores of each individual term to achieve an accurate measure of the relationship between the group of terms and their corresponding activations.

The main goal of the presented software is i) to provide a consistent set of terms by grouping them according to their meaning/function; ii) to compute new activation maps (over 1500) according to the new grouping; iii) to register individual MRIs into Montreal Neurological Institute 
Template (MNI152) [3], [4] by spatial normalization of the lesion mask, iv) visualize the lesion as well as the regions that are functionally coupled with it. With this new application, the user will be able to upload the binarized lesion and get a map of correlations (Z-score converted) with specific terms associated with the region of the lesion as with their functionally coupled regions. Moreover, two kind of visualizations are provided. One in which the user will be able to look at the brain slice by slice and inspect the depth of the lesion, and a second one where the user will be able to see in 3D the aforementioned functionally coupled regions in relation to the place of the mask.

With the presented improvements, it is expected that the users (neuropsychologists, neurosurgeons, researchers, etc.) will be able to predict the induced deficits by a potential lesion, or to better plan a forthcoming brain resection by having an extra clue about the functional role of the regions that are going to be resected. As a basic validation step, the herein proposed software was tested on seven stroke patients, predicting accurately the $91 \%$ of the measured deficits found during the neuropsychological examination.

\section{METHODS}

\section{A. Spatial Normalization}

Each human has different shape and size of brain. This is a common problem in MRI processing, when we need to make a population-wide assessment. The solution of this problem is called Spatial Normalization. In this procedure, we transform an individual MRI onto a common space called template [3], [4].

Spatial Normalization is an image registration problem between two 3D structures. The image registration is applied between the slices of the individual MRI and the slices of the template. For our approach, we used the MNI152 template [5], [6], [7], [8], [9] embedded in Neurosynth [2].

The transformation from the individual MRI to MNI152 was achieved with the affine transform [3], [4]. An affine transformation can be defined as an independent translation, rotation, scaling and sharing. In our approach, we needed a region of interest (ROI) of individual MRI to be normalized in MNI152. This can be achieved with the integration of the ROI onto individual MRI. After the image registration, we produce a new mask from registered MRI. This mask will be used during the next steps. Of relevance, the spatial normalization is applied only when the user's brain mask is not in MNI152. Under other conditions, spatial normalization step is skipped.

\section{B. Activation and Co-activation maps}

After the spatial Normalization, the ROI is aligned with the coordinates of the Neurosynth platform. Once achieved this step, Neurosynth produces co-activation maps that display the other brain regions that are activated together in similar published fMRI studies with a given, according to published studies [2]. These values are represented as Zscores (see below how Z-score is computed) which quantify the association between the presence or absence of activation in a voxel with the presence or absence of activation in a given ROI [2].

\section{Correlation between ROI and terms - Neurosynth}

Our ultimate goal is to connect a given brain region with a set of brain functions (terms), exploiting Neurosynth platform [2]. The terms presented in the Neurosynth's database are cited in different studies and the brain coordinates are in standard MNI152. More specifically, for a defined region and a study $i$, we find the regional overlap $X_{i}$ and the vector. Neurosynth calculates the matrix: $Y_{\text {Studies-Terms }}$ where $Y_{i, j}$ is the proportion of the $j^{\text {th }}$ study.

The association of $X_{i}$ with the term $\mathrm{j}$ is then defined as the Pearson correlation for the $X, Y_{i}$ as follows in (1).

$$
r_{j}=\hat{X} \widehat{Y}_{J}=\frac{\sum\left(X_{i}-\bar{X}\right)(Y-\bar{Y})}{\sqrt{\sum\left(X_{i}-\bar{X}\right)^{2}} \sqrt{\sum(Y-\bar{Y})^{2}}}
$$

After the calculation of the Pearson correlation coefficient, Fisher transform is applied [10] and a Z-score is calculated.

Considering that the terms of the Neurosynth platform are a result of text mining algorithms, the terms may include words that are not associated with brain functions or words with the same meaning. In order to solve the first obstacle, we removed the terms from the dictionary that are not relevant to brain functioning, and in order to avoid the semantic duplicates, we formed groups with terms that had the same meaning. The grouping was performed after the correlation between ROI and the different terms, so the grouping consisted of terms and their respective Z-scores.
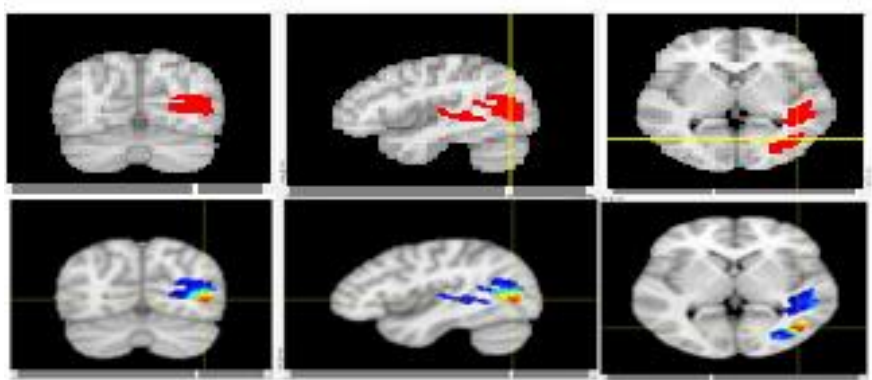

Fig. 1. In this figure, a brain mask (the first three images) is presented and its functional map for the term 'Visual' (the second three images)

As we only could return one representative Z-score for each group, we used the Stouffer's method [11] to combine all the groups' Z-scores and produce a representative Zscore:

$$
Z_{\text {total }}=\frac{\sum_{i=1}^{k} Z_{i}}{\sqrt{k}}
$$

where $Z_{\text {total }}$ is the representative $\mathrm{Z}$-score, $Z_{i}$ is the $\mathrm{Z}$ score of each member term of the group and $k$ is the number of the members of the group.

\section{Functional maps for each significant term}

Functional maps are heat maps for each of the ten highest associated terms with ROI. They represent high association (red) and low association (blue) areas for a certain term. For their construction, the first ten terms with the highest Z-scores were used, then we calculated a Zscore for each voxel in the mask and for each one of the 


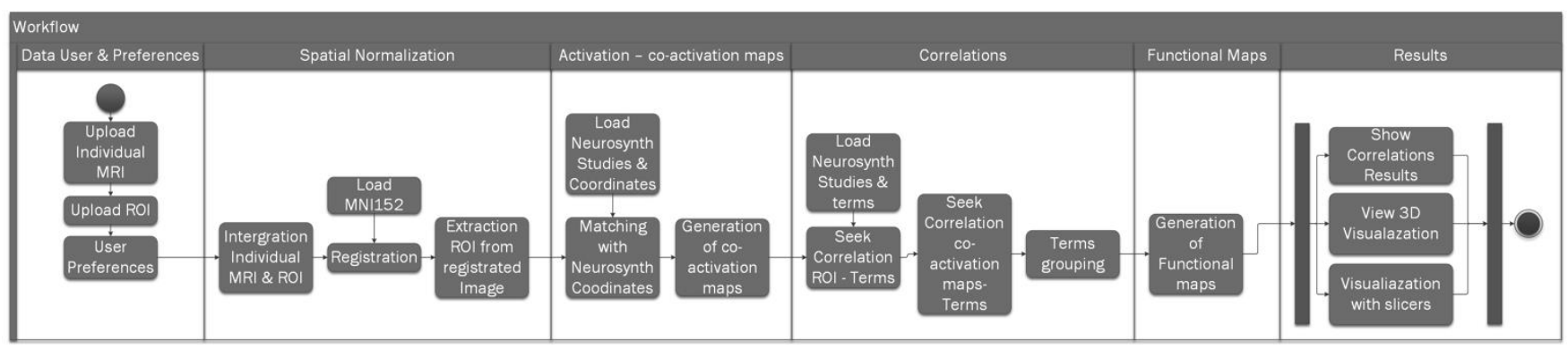

Fig. 2. In this image, we present the workflow of our project. Each box is a part of methodology and it contains functions. These functions are responsible to achieve this methodology part. Firstly, user uploads his/her data and preferences. After uploading, spatial normalization is applied to the mask Then, there must be an a match between Neurosynth coordinates and the user's data and the coactivation maps are generated After this step, the correlations between mask/coactivations maps and terms are found. In addition, functional maps are constructed about the significant terms. Finally, the results are returned to the user's computer.

aforementioned terms and, finally this Z-score map was saved and depicted. An example of functional map is shown in Fig.1.

\section{E. Validation of the proposed software}

In order to validate the proposed software employed seven ischemic stroke patients admitted to the stroke unit of the Germans Trias I Pujol University Hospital (Badalona, Spain). MRI acquisition as well as the neuropsychological examination are described in [12]. The brain MRI lesion masks were manual traced and spatial standardized by a person with experience in lesion manual tracing (RDA). The cognitive assessment was performed during the first $72 \mathrm{~h}$ after the stroke. The evaluation was accomplished for the fifteen terms with the highest Z-score and it consists of not assessed terms, correlated terms and not correlated terms.

Not-assessed terms means that these terms were not assessed during the neuropsychological assessment. Therefore, we could not test the existence of a correlation between the brain mask and the not-assessed terms due to the goals of the project and in which those patients were included.

\section{SOFTWARE DEVELOPMENT}

\section{A. Software architecture}

Client - server architecture [13] was used for our system development. The user browses the data and then selects the preferred kind of processing through the Graphical User Interface of the Desktop App (Client). Then, the data and the preferences of the user are transferred to the remote server in which the data is processed (Server). The results are transferred and presented to the user's computer (Client). The different steps of the process are being presented in Fig.2.

\section{B. Server-Side}

The package for the processing of the user's data was developed in python. This package includes image registration pipelines, as preprocessing algorithms for the preparation of the data for the next stage. In the processing stage, the cross-correlation between the ROI and the terms is calculated, as the co-activation maps and the functional maps. The structure of this package is shown in Fig.3.

The developed package can be used independently from the desktop application by the following command:

\section{python process.py folderName, ROIname, MRIname, registrationFlag, coctivationFlag, generationFunctionalMapsFlag}

where folderName is the name of the folder in which the package will save the results, ROIname is the name of the ROI file, MRIname is the name of the individual MRI (if registrationFlag=1), registrationFlag is 1 if spatial normalization is needed otherwise it's 0 , coactivationFlag is a flag with 1 if the co-activation maps are needed otherwise it's 0 and generationFunctionalMapsFlag is a flag with 1 if the functional maps are needed otherwise it's 0 .

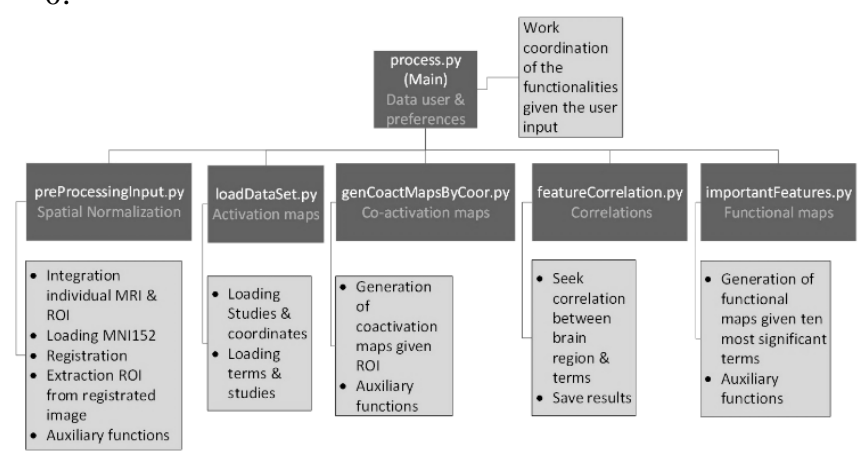

Fig. 3. This is the structure of our python package in the server. Each dark box is the source code for each methodology part and its functions are described in light box.

\section{Client-Side}

A Graphical User Interface (GUI) was developed in MATLAB and it serves as an interface of the desktopApp and the toolbox.

More specifically, client side uploads the user's data and the preferences to the server. After that, it downloads locally the results from the server. Then, it makes term groups as it was mentioned above. When the previous procedure is accomplished, it loads the arrays with the Zscores and terms, according to the previous grouping methodology. Finally, it makes the visualization according to user's options. In term of visualization, two methods have been implemented inside our software. The first one was based on "Tools for NIfTI and ANALYZE image" [https://uk.mathworks.com/matlabcentral/fileexchange/87 97-tools-for-nifti-and-analyze-image]. The second one was based on BraiNetViewer [https://uk.mathworks.com/matlabcentral/fileexchange/68 881-brainnet-viewer] [14]. 
In the case that a correlation between ROI and terms is found, the GUI provides data arrays with Z-scores and terms and images according to the two visualization methods described above. In the case of co-activation maps, we provide data array and the visualization but we have made a spinner component where the user defines a threshold of importance for the Z-score. After that, the user can select the preferred visualization to show the thresholded co-activation region. Finally, in the case of the generation of the functional maps, the user is asked to choose one of the most significant terms from a drop-down list and then the slicer visualization is opened.

\section{RESULTS}

From all the masks described in II.E with lesions, the software indicated 105 terms in total. This means that the patients may have a deficit in the functionalities related to the extracted terms. From the 105 terms, 70 were not assessed during the neuropsychological assessment, so we cannot conclude about them because our analysis is based on retrospective data. From the rest 35, where we actually had a neuropsychological examination, our software predicted correctly the 32 terms, which means $91 \%$ of accuracy. An example of the evaluation is shown in table I.

TABLE I. INDICATIVE RESULTS FOR ONE SUBJECT

\begin{tabular}{|l|l|l|}
\hline \multicolumn{1}{|c|}{ Term } & Z Score & Evaluation \\
\hline Sensory & 12.5284 & True \\
\hline Speaking & 11.1248 & True \\
\hline Movement & 8.6937 & True \\
\hline Music & 8.1895 & False \\
\hline Motion & 7.7476 & True \\
\hline Production & 5.081 & True \\
\hline Lingual & 5.5325 & True \\
\hline
\end{tabular}

Of notice, the association between brain regions and terms is rising as $\mathrm{Z}$-score rises. In addition, if we look at all the terms and the Z-scores and not only the fifteen highest, then the error rate does not change. This occurs because some of the associated terms are in low values of $\mathrm{Z}$-score. Therefore, if we have correlated and not correlated terms for lower Z-score, the error rate is not affected.

\section{DISCUSSION}

The results of our new application indicates that we are able to predict and characterize cognitive deficits with a good accuracy rate, something that was expected, since the correlation of brain regions with the terms is extracted after a meta-analysis of around 15.000 fMRI studies. Our next step will be to test this application with a larger and more heterogeneous sample. That is, not only more ischemic stroke patients, but also, tumor patients, traumatic brain injury patients as well as post-surgical brain patients.

We expect to have similar results and we will work towards reducing the error rate. Another future plan is to incorporate in our application other modalities like positron emission tomography (PET), single-photon emission computed tomography (SPECT), arterial spin labelling (ASL), quantitative susceptibility mapping (QSM) etc., in order to provide not only more information in relation to the brain state of the patient, but also, to understand, achieve and test pharmacological and not pharmacological treatments.

In a future version, we will implement an optimization algorithm, so as the software to be able to advice neurosurgeons, or surgical robots, by proposing a slightly lower volume for resection of the one imported (ROI mask), trying to maintain crucial functionalities of the brain after the surgical procedure. Of course, this is a long shot, but we believe that it's feasible.

\section{REFERENCES}

[1] Iturria-Medina Y, "Anatomical Brain Networks on the Prediction of Abnormal Brain States",BRAIN CONNECTIVITY vol. 3, no 1, pp. 1-21. 2013

[2] T. Yarkoni, R. Poldrack, T. Nichols, D. Van Essen, T. Wager, "Large-scale automated synthesis of human functional neuroimaging data", Nature methods, vol. 8, no. 8, pp. 665-670, 2011.

[3] Fatma El-Zahraa Ahmed El-Gamal, Mohammed Elmogy *, Ahmed Atwan, "Current trends in medical image registration and fusion", Egyptian Informatics Journal,pp. 99-124, 2016.

[4] Hugo J. Kuijf, J. Matthijs Biesbroek, Max A. Viergever, Geert Jan Biessels, Koen L. Vincken, "Registration of brain CT images to an MRI template for the purpose of lesion-symptom mapping",Multimodal Brain Image Analysis, vol. 8159, pp. 119128, 2013.

[5] Fonov, V., Evans, A. C., Botteron, K., Almli, R. C., McKinstry, R. C., Collins, D. L. \& Brain,Development Cooperative Group ( 2011). Unbiased average age - appropriate atlases for pediatric studies. NeuroImage, 54( 1), 313-327.

[6] Good, C. D., Johnstrude, I. S., Ashburner, J., Henson, R. N. A., Friston, K. J., \& Frackowiak, R. S. J. ( 2001). A voxel - based morphometric study of ageing in 465 normal adult human brains. NeuroImage, 14, 21-36.

[7] Grabner, G., Janke, A. L., Budge, M. M., Smith, D., Pruessner, J., \& Collins, D. L. ( 2006). Symmetric atlasing and model based segmentation: An application to the hippocampus in older adults. International Converence on Medical Image Computing and Computer - Assisted Intervention, 9, 58- 66.

[8] Mazziotta, J. C., Toga, A. W., Evans, A. C., Fox, P., \& Lancaster, J. ( 1995). A probabilistic atlas of the human brain: Theory and rationale for its development. NeuroImage, 2( 2), 89- 101.

[9] Mazziotta, J., Toga, A., Evans, A., Fox, P., Lancaster, J., Zilles, K., ... Mazoyer, B. ( 2001). A probabilistic atlas and reference system for the human brain: International Consortium for Brain Mapping (ICBM). Philosophical Transactions of the Royal Society B: Biological Sciences, 356( 1412), 1293- 1322.

[10] Fisher , R. A. , "On the "probable error" of a coefficient of correlation deduced from a small sample." Metron 1, pp.1 - 32, 1921.

[11] Stouffer, S., DeVinney, L., Suchmen, E. "The American soldier: Adjustment during army life." , Princeton University Press, Princeton, 1949.

[12] R. Dacosta-Aguayo, M. Grana, A. Savio, M. Fernandez-And ujar, M. Millan, E. Lopez-Cancio, C. Caceres, N. Bargallo, C. Garrido, M. Barrios, I. C. Clemente, M. Hernandez, J. Munuera, A. Davalos, T. Auer, and M. Mataro,'Prognostic Value of Changes in RestingState Functional Connectivity Patterns in Cognitive Recovery After Stroke: A 3T fMRI Pilot Study", Human Brain Mapping, pp. 38193831, 2014.

[13] Haroon Shakirat Oluwatosin, "Client-Server Model", IOSR Journal of Computer Engineering, vol. 16, pp. 67-71, 2014.

[14] Xia M, Wang J, He Y, "BrainNet Viewer: A Network Visualization Tool for Human Brain Connectomics." PLoS ONE 8(7): e68910., 2013 https://doi.org/10.1371/journal.pone.0068910 